\title{
A SLOWLY ROTATING COIL SYSTEM FOR AC FIELD MEASUREMENTS OF FERMILAB BOOSTER CORRECTORS*
}

\author{
G.V. Velev\#, J. DiMarco, D.J. Harding, V. Kashikhin, M. Lamm, P. Schlabach, M. Tartaglia, \\ J. Tompkins, FNAL, Batavia, IL 60510, USA..
}

\begin{abstract}
A method for measurement of rapidly changing magnetic fields has been developed and applied to the testing of new room temperature corrector packages designed for the Fermilab Booster Synchrotron. The method is based on fast digitization of a slowly rotating tangential coil probe, with analysis combining the measured coil voltages across a set of successive magnet current cycles. This paper presents results on the field quality measured for the normal and skew dipole, quadrupole, and sextupole elements in several of these corrector packages.
\end{abstract}

\section{INTRODUCTION}

In recent years, the Fermilab physics program has been extended into new accelerator-based neutrino experiments. Thus, the demand for high intensity proton beams has greatly increased. This demand established new requirements for the operation of the Fermilab Booster Synchrotron, especially for the beam losses, which cannot be satisfied by the existing corrector system, which dates back to the beginning of Booster operation in 1970 .

A stronger, water-cooled, six-in-one corrector package, which includes independently powered normal and skew field elements up to sextupole, was developed at Fermilab. After successfully testing two prototypes, the production of 60 corrector packages has begun. Forty eight of these will be installed in the Booster Synchrotron during the accelerator complex maintenance shutdowns in the next two years: half during the fall 2007 shutdown. These correctors will provide full control over the beam orbit from the injection to extraction energy (0.48.0 GeV). During standard operation, they have to follow the $15 \mathrm{~Hz}$ cycle of the main magnets; moreover, the normal quadrupole and normal and skew sextupole elements must swing through the full current range in $~ 1$ ms during the transition crossing. Additional information on the design and operational specifications of the package can be found elsewhere [1,2].

This paper presents the AC measurements performed on several corrector packages during powering cycles simulating actual operation. A comparison with DC measurements is presented as well.

\section{DESCRIPTION OF THE METHOD}

The AC profiles using for testing for the corrector elements are shown in Fig. 1. A typical current ramp rate

\footnotetext{
*Work supported by the U.S. Department of Energy
}

"velev@fnal.gov

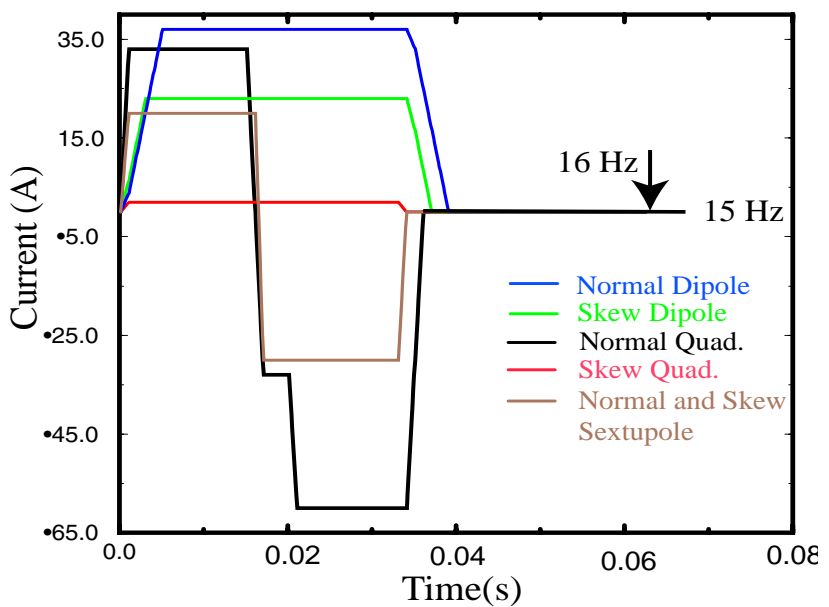

Fig. 1. Nominal current profiles for the different corrector components. The sextupole field swings from positive to negative at $50 \mathrm{kA} / \mathrm{s}$.

is on the order of several $\mathrm{kA} / \mathrm{s}$. The maximum current change of $50 \mathrm{kA} / \mathrm{s}$ is during the sextupole swing from 20 to $-30 \mathrm{~A}$ in $1 \mathrm{~ms}\left(2350 \mathrm{~T}-\mathrm{m} / \mathrm{m}^{2} / \mathrm{s}\right)$.

A typical way to measure harmonics in AC powered magnets is to utilize an array of multiple probes, similar to the one described in [3]. Such a system, which overcomes the limits to time resolution of a rotating system, has been built, and it is currently being commissioned for the final production measurements of the corrector packages.

To check the AC field quality of the first two corrector prototypes and a limited number of production magnets, a new method based on the a slowly rotating probe is utilized. This method combines measurement points from a set of successive current cycles to determine the harmonic information. A key feature is the use of the existing hardware of our fast DC rotating coil measurement system, based on a Digital Signal Processor board [4]. The technique relies heavily on the system DAQ speed.

The probe used has a standard tangential configuration of two dipole and quadrupole windings for bucking and a tangential winding for measurement of higher order harmonics [5]. To increase the resolution of our measurements, we used an encoder capable of producing 16384 triggers per rotation [6]. To synchronize the number of triggers per current cycle during the measurement, we modified the nominal Booster AC profile frequency slightly from 15 to $16 \mathrm{~Hz}$, shortening the time at zero current. (The arrow in Fig. 1 corresponds to the end of the $16 \mathrm{~Hz}$ profile.)

Fig. 2 shows the measured flux signal (black line) when the dipole corrector is excited. For this measurement, a nominal rotational probe speed of $0.25 \mathrm{~Hz}$ is used, 


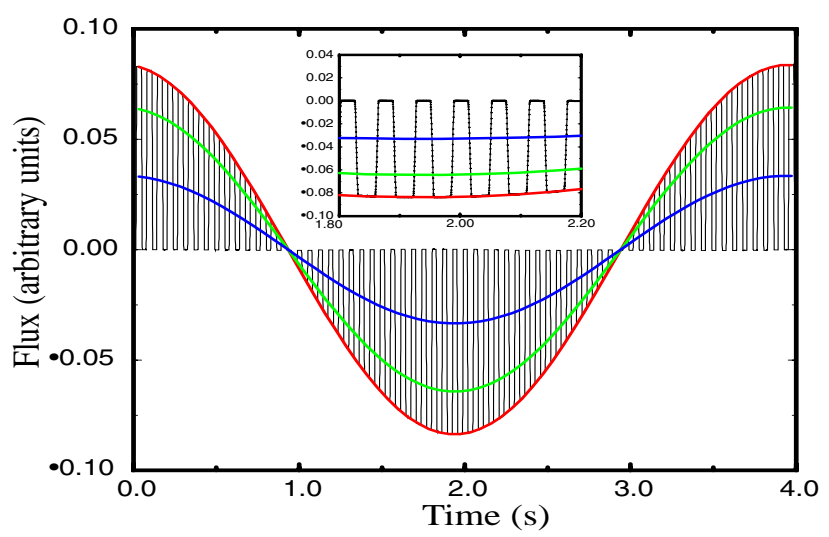

Fig. 2. Measured flux with the dipole component powered. The colored lines correspond to points at a fixed phase in time with respect to the rapidly changing dipole field. Therefore, these correspond to flux at different angles but at a fixed current. The inset shows the inflated time interval from 1.8 to $2.2 \mathrm{~s}$.

accruing 16384 flux samples per rotation, which corresponds to a time resolution of $244.1 \mu \mathrm{s}$. For one full rotation of the probe, the current cycles 256 times. Each cycle is sampled by 64 points. Selecting points with the same time phase relative to the beginning of ongoing powering cycles, one acquires flux as a function of angle at a fixed current. Field harmonics are then reconstructed by applying the standard rotating coil analysis [5]. By varying this time phase, we measure the field as a function of current. For example, three sets of points with the same probe angular phases are shown in Fig. 2 with different color sinusoidal curves.

During the initial tests, we found that we accumulate an error, in average, of $\sim 20 \mu$ s after every $4 \mathrm{~s}$ rotation due to the precision of the control unit of our stepper motor. This effect is shown in Fig. 3, where the black lines represent the measurements of the normal dipole strength after $~ 50$ probe rotations. To compensate for this systematic effect, we applied a time correction based on the difference between the expected and measured current cycles. The red curve (Fig. 3) shows the average dipole strength after the time correction.

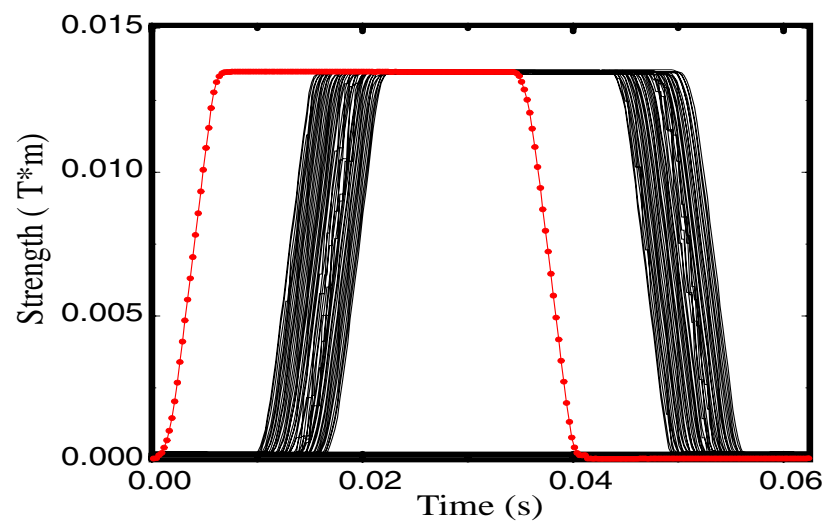

Fig. 3. Dipole strength before (black) and after (red) time correction due to the $20 \mu$ s desynchronization between the probe rotations.
Additional effects of rotational speed and power supply short and long range instabilities, as well as further discussion of the method, are presented elsewhere [7].

\section{AC MAGNETIC FIELD MEASUREMENTS}

Applying the described method, two pre-production prototypes (BMA000-001) and two production corrector packages (BMA009-010) were measured. To quantify these AC measurements, they are compared to quality DC measurements performed with the same probe and DAQ system. The comparison between the AC measured and expected strengths for normal dipole, normal and skew quadrupole, and normal sextupole are shown in Fig. 4. We do not present the skew dipole and sextupole components, by design they have the same coil configuration, rotated by 90 and 30 degrees respectively. The expected strength is calculated using the transfer functions, measured at the maximum positive DC current for the corrector components, multiplied by the current profile. One can conclude that the AC measurements are in good agreement with the expected strengths. The small discrepancy at the beginning and the end of the current profile in the skew quadrupole component (D) is due to some problem, perhaps mismatch between the regulator setting and value of the inductance load, in that particular power supply unit.

To check for possible effects of eddy currents induced during fast ramping, we compared the first allowed harmonics of the corrector elements measured with AC and DC profiles. The DC measurements are performed with a stair-step current profile, where the ramp rate between the DC steps is $\sim 0.5 \mathrm{~A} / \mathrm{s}$. The result of the comparison is shown in Fig. 5, where the plots from top to bottom are $b_{3}, b_{6}$ and $b_{9}$ [5] field harmonics (in units at 1 inch) for the dipole, quadrupole and sextupole elements in BMA001. The black circles (red squares) represent the AC (DC) measurements. Good agreement between AC
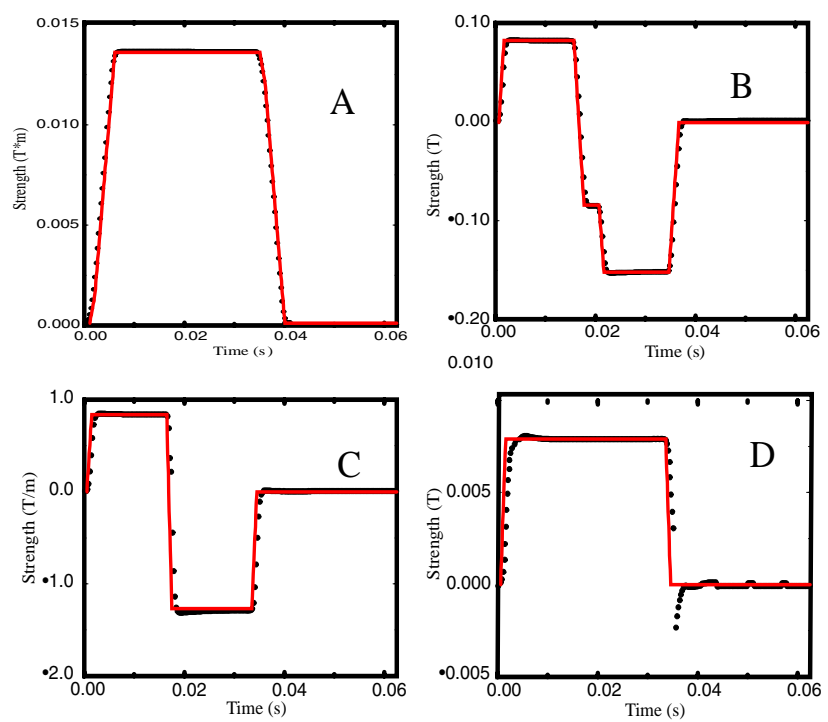

Fig. 4. Dipole (A), normal (B) and skew (D) quadrupole and sextupole $(\mathrm{C})$ corrector strengths. The points are the measured values and the lines are the expected strengths. 

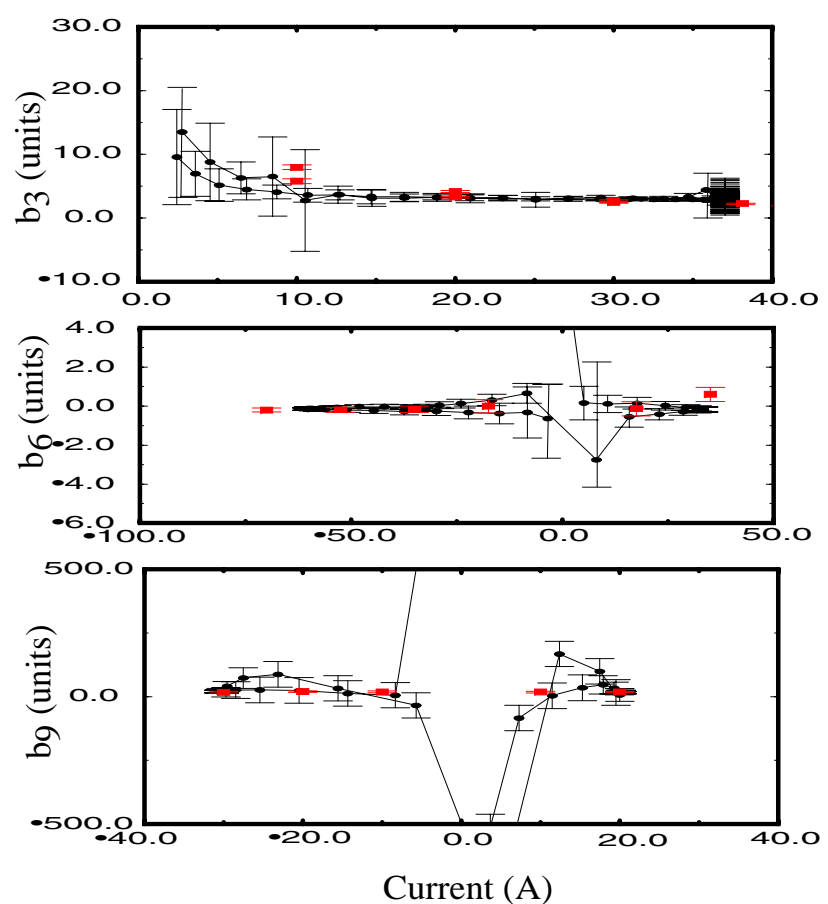

Fig. 5. The first allowed harmonics $b_{3}, b_{6}$, and $b_{9}$ in the dipole, quadrupole and sextupole corrector respectively. The black (red) points represent the AC (DC) measurements.

and DC measurements is observed which suggests small eddy current effects in the correctors. The large errors in $b_{9}$ (Fig. 5, bottom plot) are a consequence of the fact that the absolute level of the signals are comparable with the expected resolution of the method for the higher order harmonics.

In the next step, we checked for possible effects on the field from the beam position monitor (BPM). A prototype of the BPM, made from $\sim 3 \mathrm{~mm}$ thick stainless steel tube, was inserted in the magnet aperture. A typical example of the $b_{3}$ and $b_{6}$ field harmonics in the dipole and quadrupole corrector components without (black) and with (red) BPM is shown in Fig 6. We found that some of the harmonics, mostly allowed ones, are increased up to several percent from the main fields.

\section{CONCLUSION}

We proposed a new method for AC field measurements for the corrector upgrade of Fermilab Booster. The method is based on the existing, in house made [4], fast continuous rotating coil measurement system. It uses the ability to synchronize the AC current period with the rotation of the measurement probe and obtain the field harmonics connecting the points from different $\mathrm{AC}$ cycles.

In addition, we measured four BMA corrector packages, excited with $16 \mathrm{~Hz}$ AC profiles, and found good consistency with DC measurements. The comparison between the first allowed harmonics pointed toward small eddy current effects in the corrector fields.

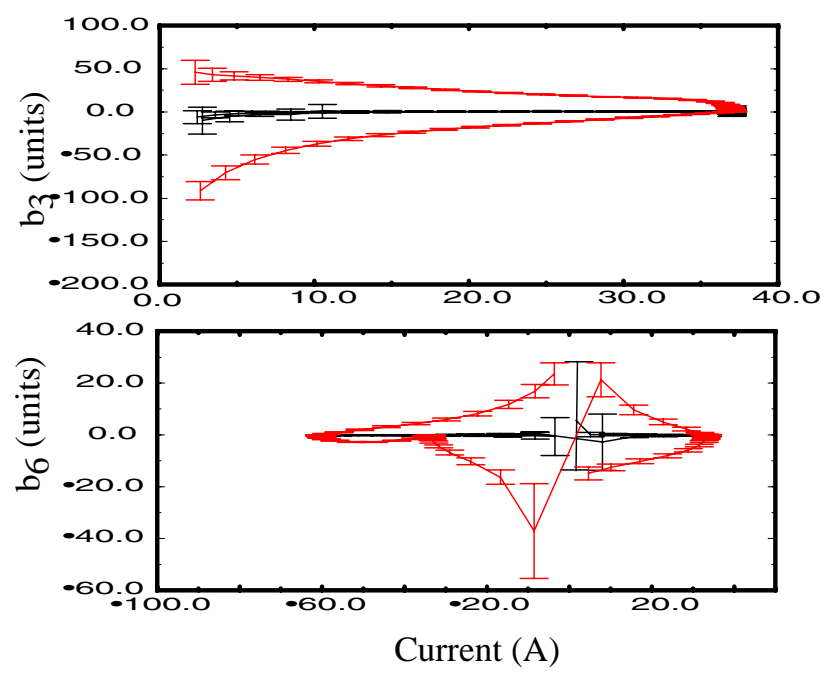

Fig. 6. The sextupole and dodecapole field harmonics in the dipole and quadrupole corrector components. The black (red) lines correspond to the measurements without (with) BPM.

We found that the inserted BPM largely increases the harmonics hysteresis width; future studies are needed with different grounding scenarios.

We proved that this method could be used to measure the harmonics in the case of fast changing fields with accuracy sufficient for the warm corrector packages.

\section{REFERENCES}

[1] V.S. Kashikhin et al., “A New Correction Magnet Package for the Fermilab Booster Synchrotron” in Proc. of 2005 PAC, Knoxville, Tennessee, pp. 12041206.

[2] D.J. Harding et al., "Design and Fabrication of a Multi-Element Corrector Magnet for the Fermilab Booster Synchrotron”, These Proceedings, MOPAS006.

[3] A. Jain et al., "Magnetic Field Measurements for Fast Changing Magnetic Fields”, IEEE Trans. Applied Superconductivity, vol. 15, no. 2, June 2005, pp 1221-1224.

[4] G.V. Velev et al., “A Fast Continuous Magnetic Field Measurement System Based on Digital Signal Processors”, IEEE Trans. of Applied Superconductivity, Vol. 16, No. 2, June 2006, pp. 1374-1377.

[5] A. Jain, "Harmonic coils”, CERN 98-05, Aug. 1998.

[6] We use an differential encoder with 4096 pulses per rotation from BEI Industrial Encoder Division, http://www.beiied.com. Using the slopes of the A and $\mathrm{B}$ encoder pulses, we generate 16384 trigger pulses for the DAQ system.

[7] G.V. Velev et al., "AC Field Measurements of Fermilab Booster Correctors Using a Rotating Coil System" in Proc. of 2006 EPAC, Edinburgh, Scotland, pp. 2574-2576. 